\title{
Photometry and spectroscopy of the central star of the Trifid nebula $^{\star}$
}

\author{
L. Kohoutek ${ }^{1}$, P. Mayer ${ }^{2}$, and R. Lorenz ${ }^{3}$ \\ 1 Hamburger Sternwarte, Gojenbergsweg 112, D-21029 Hamburg, Germany \\ 2 Astronomical Institute, Charles University, V Holešovičkách 2, 18000 Praha 8, Czech Republic \\ 3 Dr. Remeis-Sternwarte, Astronomisches Institut der Universität Erlangen-Nürnberg, Sternwartstraße 7, D-96049 Bamberg, \\ Germany
}

Received December 12, 1997; accepted July 27, 1998

\begin{abstract}
U B V$ photometry of the central star of the Trifid nebula - HD 164492, which has so far been suspected of photometric as well as of radial velocity variability - is presented. The results of our photometry do not confirm any variability. Moreover, based on new high resolution CCD spectra any radial velocity variability can be discarded. Photometry of several other members of the multiple stellar system is included; according to this photometry, the system is a physical one. Equivalent widths for the main component of HD 164492 are given. Positions measured on CCD exposures are presented too.
\end{abstract}

Key words: binaries: visual — stars: early-type - stars: HD 164492 - ISM: Trifid nebula

\section{Introduction}

The star HD 164492, of spectral type O7.5 $\operatorname{III}((\mathrm{f}))$ (Walborn 1973), is the central object of the multiple system ADS 10991. Undoubtedly it is responsible for the ionisation of the Trifid nebula (NGC 6514; M 20). According to ADS, the components of the system are: $\mathrm{A}-8.0^{\mathrm{m}}$, $\mathrm{B}-10.6^{\mathrm{m}}\left(\mathrm{AB} 6^{\prime \prime}\right), \mathrm{C}-8.8^{\mathrm{m}}\left(\mathrm{AC} 11^{\prime \prime}\right), \mathrm{D}-10.5^{\mathrm{m}}(\mathrm{CD}$ $\left.2^{\prime \prime}\right), \mathrm{E}-12.4^{\mathrm{m}}\left(\mathrm{CE} 6^{\prime \prime}\right), \mathrm{F}-13.8^{\mathrm{m}}\left(\mathrm{AF} \mathrm{22^{ \prime \prime }}\right)$ and $\mathrm{G}-$ $13.2^{\mathrm{m}}$ (CG 30"). Gahm et al. (1983) classified component $\mathrm{B}$ as A2 Ia, component $\mathrm{C}$ as B6 V and E as F3 V. The star was measured photoelectrically many times, however, the results of photometry differed. Therefore it was added to the New Catalogue of Suspected Variable Stars (Kukarkin et al. 1982) as NVS 10013. According to the spectroscopic data so far available, the radial velocities of components $\mathrm{A}$ and $\mathrm{C}$ are variable, too (see e.g. Abt \& Biggs 1972).

Send offprint requests to: P. Mayer

* Based on observations collected at the European Southern Observatory, La Silla, Chile.
Table 1 collects the published photometry of this star. Remarkable is the note by Borgman (1960) about the difference between his two measurements; it can be noted that another star for which Borgman gives similarly different data - HD 35921 - was later proved to be an eclipsing binary (LY Aur). In case of HD 164492, some differences in photometry may result from the diaphragm used: a different amount of the bright nebulosity as well as further components may enter the diaphragm. Unfortunately, the diaphragm diameters are rarely indicated by the authors, so it is difficult to judge the effects mentioned. The system was measured by HIPPARCOS (ESA 1997), however, the photometry has a large scatter due to the multiplicity (HIPPARCOS magnitudes are 7.5, 10.4 and 8.9 for components $\mathrm{A}, \mathrm{B}$ and $\mathrm{C}$, respectively).

\section{Photometry}

In order to inspect whether the main star (comp. A) is variable or not, we measured it in 30 nights during 6 observing periods (1985 to 1992) at the European Southern Observatory, La Silla, Chile. The measurements were made in the $U B V$ system with the Bochum $61 \mathrm{~cm}$ telescope and the ESO $50 \mathrm{~cm}$ telescope. Other components were measured too. The results for components $\mathrm{AB}$ are given in Table 2 and for the other components in Table 3.

One can see that the measurements by Lindroos (A, CD) agree well with our data. The measurement by Rufener $(\mathrm{AB})$ is also close to our value, however the discrepancies of the earlier data remain unexplained. In particular, we cannot agree with magnitudes and colours as given by Echevarria et al. Using values from Table 3, integral magnitudes of various component combinations can be calculated ( $V$ magnitudes follow):

AB: 7.538, ABCD: 7.208, CDE: 8.621, ABCDE: 7.197. 
Table 1. Previously published $U B V$ data for HD 164492 (visual magnitudes similar to $V$ system are also listed)

\begin{tabular}{lcccl}
\hline Source & $V$ & $B-V$ & $U-B$ & Note \\
\hline Pickering (1908) & 6.91 & & & visual photometry \\
Hiltner (1956) & 7.63 & 0.00 & -0.86 & \\
Borgman (1960) & 8.16 & & & two measurements made in two nights \\
& 8.26 & & & \\
Klare \& Neckel (1977) & 7.30 & 0.02 & -0.83 & $30^{\prime \prime}$ diaph., probably comp. ABCDE \\
Echevarria et al. (1979) & 7.24 & -0.11 & -0.95 & $27^{\prime \prime}$ diaph., comp. AB \\
& 7.38 & 0.19 & -0.25 & $27^{\prime \prime}$ diaph., comp. CDE \\
& 13.17 & 0.17 & -0.03 & $27^{\prime \prime}$ diaph., comp. G \\
Rufener 1981 & 7.517 & & & double star, not separated \\
Lindroos (1983) & 7.593 & & & component A \\
& 8.671 & & & components CD \\
\hline
\end{tabular}

Table 2. $U B V$ photometry - components $\mathrm{AB}$

\begin{tabular}{crrrrr}
\hline Date & $V$ & $B-V$ & $U-B$ & $n$ & $T^{a}$ \\
yymmdd & & & & & \\
\hline 850514 & 7.576 & -.011 & & 2 & 1 \\
850515 & 7.542 & +.002 & & 3 & 1 \\
850516 & 7.542 & .000 & & 2 & 1 \\
850521 & 7.543 & -.004 & & 1 & 1 \\
& & & & & \\
860420 & 7.534 & -.003 & & 2 & 1 \\
860421 & 7.545 & +.002 & & 4 & 1 \\
860424 & 7.529 & -.001 & & 13 & 1 \\
860429 & 7.535 & -.001 & & 3 & 1 \\
& & & & & \\
860430 & 7.520 & +.023 & & 2 & 2 \\
860501 & 7.537 & -.008 & & 4 & 2 \\
860504 & 7.510 & +.001 & & 4 & 2 \\
860505 & 7.541 & -.008 & & 4 & 2 \\
860507 & 7.528 & +.004 & & 4 & 2 \\
860508 & 7.538 & +.004 & & 4 & 2 \\
& & & & & \\
870423 & 7.582 & +.005 & & 2 & 2 \\
870426 & 7.526 & +.003 & & 2 & 2 \\
870427 & 7.519 & -.003 & & 2 & 2 \\
870430 & 7.519 & +.007 & & 2 & 2 \\
870503 & 7.508 & +.005 & & 2 & 2 \\
870509 & 7.536 & -.012 & & 2 & 2 \\
& & & & & \\
900417 & 7.542 & -.006 & -.91 & 3 & 2 \\
900428 & 7.556 & -.012 & -.91 & 2 & 2 \\
900430 & 7.552 & +.003 & -.90 & 3 & 2 \\
& & & & & \\
920301 & 7.540 & -.002 & -.91 & 2 & 2 \\
920302 & 7.535 & +.003 & -.91 & 2 & 2 \\
920303 & 7.564 & -.008 & -.92 & 2 & 2 \\
920304 & 7.580 & +.001 & -.91 & 2 & 2 \\
920305 & 7.551 & -.002 & -.91 & 2 & 2 \\
920306 & 7.567 & +.014 & -.90 & 2 & 2 \\
920308 & 7.544 & +.002 & -.92 & 2 & 2 \\
\hline & & & & &
\end{tabular}

${ }^{a}$ Telescope: 1 - Bochum $61 \mathrm{~cm}\left(\mathrm{dia}=18.2^{\prime \prime}\right)$, 2 - ESO $50 \mathrm{~cm}\left(\mathrm{dia}=20.9^{\prime \prime}\right)$.
The multiple system is located in the brightest, albeit homogenous, part of the nebula. The components C, D and $\mathrm{E}$ are rather close to one of the well-known dark lanes (see e.g. Lynds et al. 1985). The measurements were made with small diaphragms and the sky was measured in points close to the system; then the effect of the background brightness on measurements of the components $\mathrm{AB}$ can be estimated as not larger than about $0 . \mathrm{m} 01$. Nevertheless, the scatter of our measurements of the $B$ and $V$ magnitudes in individual nights is slightly larger than expected. The $U$ data obtained in the observing epochs 1986 and 1987 show a rather large scatter, which cannot completely be understood. Probably, the reason is a non-sufficient number of measurements of standard stars with strongly negative $U-B$ index, which caused some uncertainties during the reduction procedure. Hence, these $U$ data were discarded.

The $U B V$ magnitudes of individual components (Table 3) were determined from measurements made in two observing periods (1985-86) - in nights with good seeing - using the Bochum $61 \mathrm{~cm}$ telescope with small diaphragm (diameter 11.3" ${ }^{\prime \prime}$. The sky was measured in two or four points located close to and symmetrically around the stars. In spite of this, the data for fainter components might be affected by the nonhomogenous background.

We made also CCD observations with the $2.2 \mathrm{~m}$ telescope at ESO, La Silla, in April 1986. In order to diminish the brightness of the surrounding nebula, we used the IHW cometary filter C2 with the central wavelength $513.6 \mathrm{~nm}$ and FWHM $9.0 \mathrm{~nm}$. Two frames with integration times $15 \mathrm{~s}$ and $8 \mathrm{~s}$ were taken; the A component is saturated on both frames. The $\mathrm{C} 2$ intensities are given in Table 3 (intensity of $\mathrm{G}$ component was set to 10.0). The positions of the components are shown in Fig. 1 and given in Table 4. They are nearly identical with values as given in ADS.

From the dereddened colour indices $(B-V)_{0}$ it appears that the brighter components $\mathrm{A}$ to $\mathrm{E}$ are of early spectral type and probably form a physical system - the nucleus of the nebula. However, these dereddened indices disagree with the classification by Gahm et al. (1983) mentioned in Introduction. On the other hand, Lindroos 1983) gives 
Table 3. $U B V$ photometry of individual components

\begin{tabular}{ccccccc}
\hline Component & $V$ & $B-V$ & $U-B$ & $(B-V)_{0}$ & $E_{B-V}$ & $I(\mathrm{C} 2)$ \\
\hline $\mathrm{A}$ & 7.610 & -.010 & -.973 & -.31 & 0.32 & satur. \\
$\mathrm{B}$ & $10.52:$ & $+.12:$ & $-.58:$ & -.22 & 0.34 & 127.7 \\
$\mathrm{CD}$ & 8.660 & +.084 & -.748 & -.27 & 0.35 & $653.9^{a}$ \\
$\mathrm{E}$ & 12.24 & +.20 & -.58 & -.24 & 0.44 & $13:^{b}$ \\
$\mathrm{~F}$ & 13.96 & +.63 & +.06 & & & \\
$\mathrm{G}$ & 13.35 & +.64 & $+.77:$ & & & 10.0 \\
\hline
\end{tabular}

${ }^{a}$ The components are very close but it is possible to separate them using a small diaphragm; C2 intensity ratio $I(\mathrm{C}) / I(\mathrm{D})$ is 3.9/1.0. ${ }^{b}$ The bright components $\mathrm{C}+\mathrm{D}$ are in close vicinity.

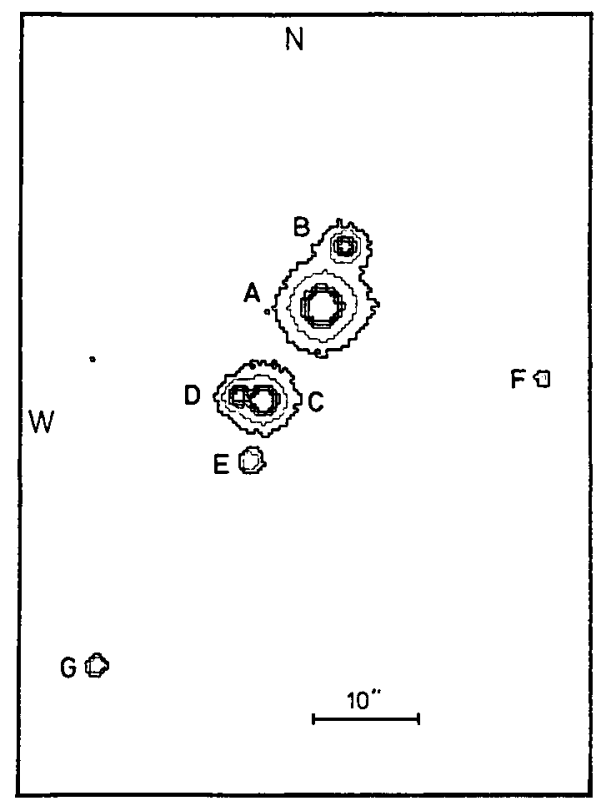

Fig. 1. Central part of nebula M 20 (Trifid). $2.2 \mathrm{~m}$ telescope + CCD at ESO, La Silla. IHW cometary filter C2, April 1986

Table 4. Positions of individual components of HD 164492 (relative to component $\mathrm{A}$; in footnotes, positions relative to component $\mathrm{C}$ are given)

\begin{tabular}{lrrc}
\hline Component & $\begin{array}{r}d \\
\prime \prime\end{array}$ & $\begin{array}{c}\mathrm{PA} \\
\circ\end{array}$ & $\begin{array}{c}\mathrm{D} \\
\mathrm{AU}\end{array}$ \\
\hline $\mathrm{B}$ & 6.22 & 20.7 & 14300 \\
$\mathrm{C}$ & 10.65 & 212.3 & 24500 \\
$\mathrm{D}^{a}$ & 11.74 & 223.2 & 27000 \\
$\mathrm{E}^{b}$ & 16.38 & 204.9 & 37650 \\
$\mathrm{~F}$ & 22.01 & 108.1 & \\
$\mathrm{G}^{c}$ & 40.51 & 212.3 & \\
\hline
\end{tabular}

${ }^{a} d=2.39^{\prime \prime}, \mathrm{PA}=280.8^{\circ}$

${ }^{b} d=5.98^{\prime \prime}, \mathrm{PA}=191.6^{\circ}$

${ }^{c} d=29.86^{\prime \prime}, \mathrm{PA}=212.3^{\circ}$.
Table 5. Positions and C2 intensities of surrounding stars (relative to HD 164492 component A)

\begin{tabular}{rccc}
\hline $\begin{array}{r}\text { Star } \\
\text { No. }\end{array}$ & $\begin{array}{c}\text { delta } \\
\alpha\end{array}$ & $\begin{array}{c}\text { delta } \\
\beta\end{array}$ & $\begin{array}{c}I(\mathrm{C} 2) \\
{[G=10]}\end{array}$ \\
\hline 1 & -2.8 & +79.4 & 2.65 \\
2 & +25.5 & +74.3 & 15.3 \\
3 & $+32.0:$ & $+8.3:$ & 0.35 \\
4 & -4.1 & +16.2 & 0.31 \\
5 & -5.9 & +6.4 & 1.28 \\
6 & $-13.6:$ & $-8.5:$ & 0.85 \\
7 & -43.1 & -63.0 & 2.34 \\
8 & -40.5 & -75.9 & 2.21 \\
9 & -34.5 & -82.2 & 3.41 \\
10 & $+43.6:$ & $-45.3:$ & 0.60 \\
\hline
\end{tabular}

uvby values for $\mathrm{CD}$, which also correspond to a spectral type of about B0.5, as our $U B V$ values do.

We also measured positions (relative to the component A) and C2 intensities (supposing intensity of G 10.0) of several stars in the field believing that this can be useful for further investigation. The results are given in Table 5 .

\section{Spectroscopy}

Since the radial velocity of the A component was reported as variable in three references - data in literature varies from -32 to $+10.5 \mathrm{~km} \mathrm{~s}^{-1}$ - we also took several CCD spectra with the coudé echelle spectrograph ECHELEC attached to the $1.52 \mathrm{~m}$ ESO telescope. The CCD chip RCA \#13 (known to be affected by few defect pixel rows) was used. The average linear dispersion was $3.8 \AA \mathrm{mm}^{-1}$, the slit width was in the range $1.4^{\prime \prime}$ to $2.5^{\prime \prime}$, equivalent to a resolving power of 23000 to 32000 . The useful wavelength range was about $300 \AA$, and various central wavelengths were chosen. The reduction of the spectra was performed with the Munich Image Data Analysis System (MIDAS). For the determination of radial velocities and equivalent widths, the spectral lines He I 4471, N II 4481, N III 4511, HeII 4541, Si IV 4631, C III 4647, Si IV 4654, He II 4686, He I 4713 and He I 4922 were fitted with single Gaussian 
Table 6. Heliocentric radial velocities from ECHELEC spectra (in $\mathrm{km} \mathrm{s}^{-1}$ ) for HD 164492 (ADS 10991 A)

\begin{tabular}{|c|c|c|c|c|c|c|c|c|c|c|c|c|}
\hline $\begin{array}{c}\text { HJD } \\
-2440000\end{array}$ & $\begin{array}{r}\mathrm{He} \mathrm{I} \\
4471\end{array}$ & $\begin{array}{r}\mathrm{N} \text { II } \\
4481\end{array}$ & $\begin{array}{l}\text { N III } \\
4511\end{array}$ & $\begin{array}{l}\text { He II } \\
4541\end{array}$ & $\begin{array}{l}\text { Si IV } \\
4631\end{array}$ & $\begin{array}{l}\text { C III } \\
4647\end{array}$ & $\begin{array}{l}\text { Si IV } \\
4654\end{array}$ & $\begin{array}{l}\text { He II } \\
4686\end{array}$ & $\begin{array}{r}\text { He I } \\
4713\end{array}$ & $\begin{array}{r}\mathrm{He} \mathrm{I} \\
4922\end{array}$ & $\begin{array}{r}\text { Mean } \\
\text { all lines }\end{array}$ & $\begin{array}{l}\text { Mean } \\
\text { He I,II }\end{array}$ \\
\hline 8442.698 & -2.4 & & & 3.4 & -22.1 & -14.3 & 10.2 & & -2.3 & & 1.3 & -0.4 \\
\hline 8459.737 & 1.0 & 4.8 & 4.7 & 7.2 & -4.9 & -5.6 & 9.9 & 3.2 & & & 2.5 & 3.8 \\
\hline 8674.870 & & & & & -6.7 & 3.8 & 23.1 & 3.8 & -0.6 & & 4.7 & 1.6 \\
\hline 8676.871 & & & & & -3.6 & -13.8 & 9.4 & 1.7 & -2.7 & & -1.8 & -0.5 \\
\hline 8678.873 & & & & & -3.9 & -3.8 & 4.0 & 2.8 & 2.8 & & 0.4 & 2.8 \\
\hline 8762.845 & & & & & & -5.0 & & 4.0 & 2.7 & & 0.6 & 3.4 \\
\hline 8763.843 & & & & & & -0.2 & 3.6 & 1.0 & 1.0 & 2.5 & 1.6 & 1.5 \\
\hline Average m.e. & 1.9 & 2.1 & 5.6 & 4.4 & 3.1 & 4.2 & 4.4 & 1.3 & 1.8 & 1.1 & 0.8 & 0.7 \\
\hline Average vel. & & & & & & & & & & & 1.3 & 1.7 \\
\hline
\end{tabular}

profiles, using the Newton-Raphson method to optimize the central wavelength, the full width at half-maximum and the amplitude of the lines. For the line He I 4471, which is a blend of two components, a weighted average 4471.508 was used as the reference table wavelength.

Figure 2 shows two typical merged ECHELEC-spectra of HD 164492 (A). The most prominent lines are indicated, and features, which are due to the CCD defects, are marked as "CCD". Table 6 represents the radial velocities, and in Table 7 the measured equivalent widths are given. The most accurate radial velocities result from He II 4686 (its average value is $+2.8 \pm 0.4 \mathrm{~km} \mathrm{~s}^{-1}$ ), which is the strongest helium line in the spectra.

Our results do not confirm any radial velocity variability of the main component A of HD 164492 (A). It should be stressed that in many cases low-dispersion spectra of OB stars yield imaginary radial velocity variations, which cannot be confirmed by modern measurements using high dispersion spectra (see e.g. Mayer et al. 1994).

\section{Conclusions}

As noted in Sect. 2, the system is probably a physical one (contrary to the conclusion by Lindroos 1985). Using values as given in Table 3, $V$ magnitudes can be corrected for extinction. In case of the CD components, $(B-V)_{0}$ for the $\mathrm{C}$ component was calculated taking the expected colour of the D component into account. Note that among determinations of the distance to M 20 there is some discrepancy in the data in literature. E.g., Stone (1978) gives $2.84 \mathrm{kpc}$, Avedisova \& Kondratenko (1984) $1.95 \mathrm{kpc}$, Lynds et al. (1985) $1.68 \mathrm{kpc}$. With $M_{V}$ values according to SchmidtKaler (1982) and $A_{V}=3.1 E(B-V)$, an average value of the distance modulus for the $\mathrm{A}, \mathrm{B}, \mathrm{C}$ and $\mathrm{E}$ components is $12.2^{\mathrm{m}}$, i.e., the distance equals $2.8 \mathrm{kpc}$. If $A_{V}=1.3 \mathrm{ac}-$ cording to Lynds et al. (1985), then the distance is $2.5 \mathrm{kpc}$.

No variability, photometric or spectroscopic, has been found for the star HD 164492 (ADS $10991 \mathrm{~A}$ ). If there is any variability, then it is certainly less than announced in earlier papers, and less than our measuring errors about \pm 0.04 in magnitude $(V)$ or $\pm 1 \mathrm{~km} \mathrm{~s}^{-1}$ in radial velocity. Note also, that our investigation on HD 164492 shows the urgence to use high dispersion spectra for the radial velocity determination of $\mathrm{OB}$ stars.

Table 7. Equivalent widths for HD 164492 (ADS 10991 A) in $\AA$ derived from ECHELEC spectra

\begin{tabular}{lccc}
\hline Line & $\begin{array}{c}\text { EW } \\
\AA\end{array}$ & $\begin{array}{c}\delta\left(\mathrm{EW}^{a}{ }^{a}\right. \\
\AA\end{array}$ & $\begin{array}{c}\text { Number of } \\
\text { measurements }\end{array}$ \\
\hline He I 4471 & 0.426 & 0.023 & 2 \\
N II 4481 & 0.072 & - & 1 \\
N III 4511 & 0.108 & - & 1 \\
He II 4541 & 0.626 & 0.143 & 2 \\
Si IV 4631 & 0.094 & 0.009 & 5 \\
C III 4647 & 0.140 & 0.021 & 7 \\
Si IV 4654 & 0.075 & 0.033 & 7 \\
He II 4686 & 0.714 & 0.034 & 6 \\
He I 4713 & 0.187 & 0.025 & 6 \\
H $\beta$ & 2.243 & 0.138 & 2 \\
He I 4922 & 0.270 & - & 1 \\
\hline
\end{tabular}

${ }^{a}$ standard deviation relative to averaged value.

\section{References}

Abt H.A., Biggs E.S., 1972, Bibliography of Stellar Radial Velocities, New York, p. 365

Avedisova V.S., Kondratenko G.I., 1984, Naucnye Informacii 56,59

Borgman J., 1960, BAN 15, 255

Echevarria J., Roth M., Warman J., 1979, Rev. Mex. Astron. Astrofiz. 4, 287

ESA (European Space Agency), 1997, The Hipparcos and Tycho Catalogues, ESA SP-1200, ESA/ESTEC Noordwijk Gahm G.F., Ahlin P., Lindroos K.P., 1983, A\&AS 51, 143

Hiltner W.W., 1956, ApJS 2, 387

Klare G., Neckel Th., 1977, A\&AS 27, 215 

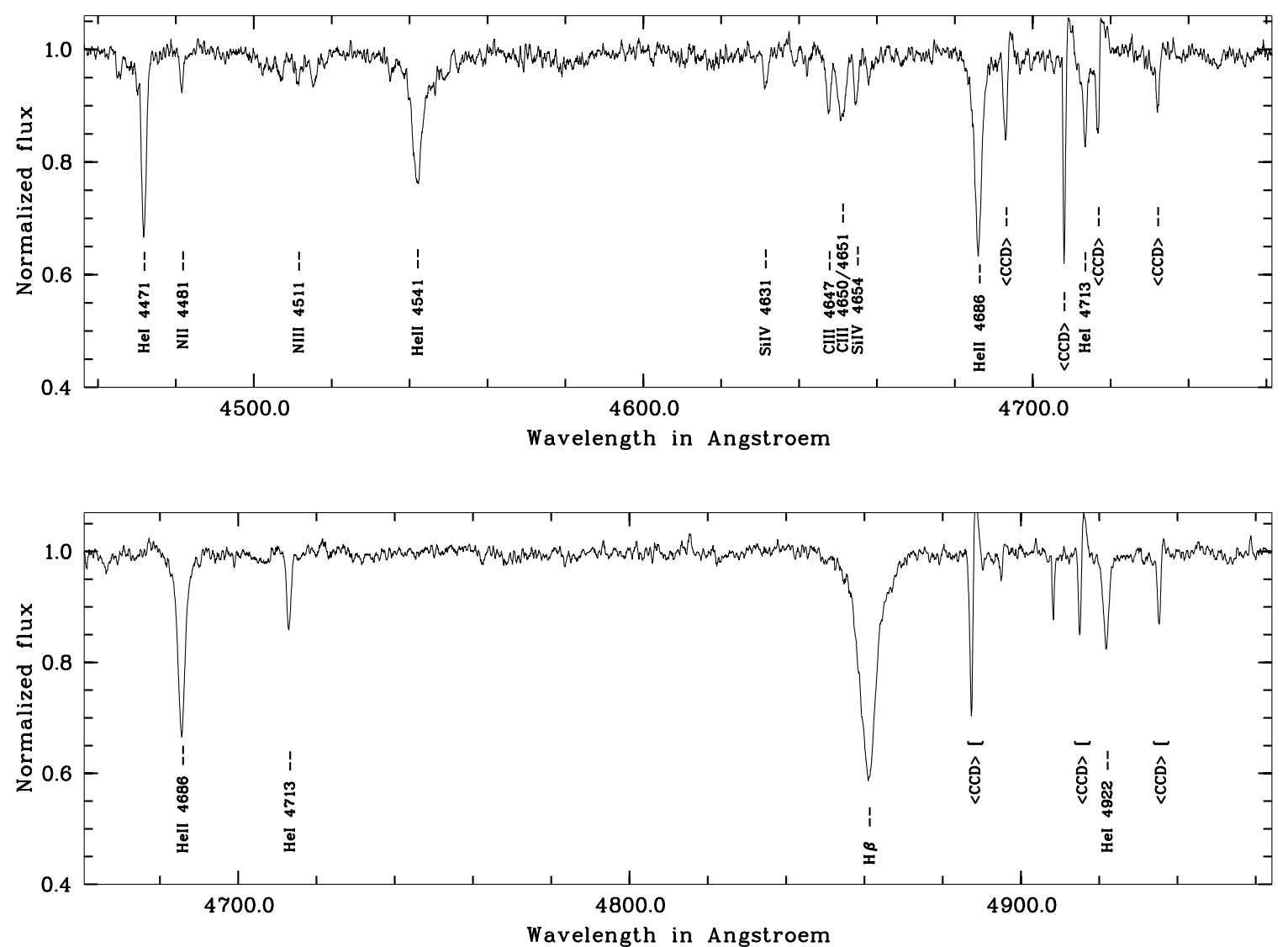

Fig. 2. Typical merged ECHELEC spectra of HD 164492 (component A) taken at hel. JD 2448459.7366 (above) and 2448763.8435 (below). Some prominent lines are indicated, features caused by a CCD defect are marked as $<$ CCD $>$

Kukarkin B.V., et al., 1982, New Catalogue of Suspected Variable Stars, Nauka, Moscow

Lindroos K.P., 1983, A\&AS 51, 161

Lindroos K.P., 1985, A\&AS 60, 183

Lynds B.T., Canzian B.J., O'Neil Jr. E.J., 1985, ApJ 288, 164

Mayer P., Chochol D., Hanna M.-A., Wolf M., 1994, Contrib. Astron. Obs. Skalnate Pleso 24, 65
Pickering E.C., 1908, Harvard Annals 54, 1

Rufener F., 1981, A\&AS 45, 207

Schmidt-Kaler, T., 1982, in: Schaifers K., Voigt H.H. (eds.), Landolt-Börnstein VI/2b. Springer, Berlin

Stone R.C., 1978, AJ 83, 393

Walborn N.R., 1973, AJ 78, 1067 